\title{
Classically conditioned bradycardia and skeletal-motor activity in restrained rats
}

\author{
ROBERT D. FITZGERALD, GENE STAINBROOK, and DAVID FRANCISCO \\ Oregon Health Sciences University, Portland, Oregon
}

\begin{abstract}
Classical conditioning of heart rate (HR) and skeletal-motor activity in restrained rats was examined using a single, brief (.5-msec) pulse of shock to the rat's tail as both the CS and US. Electric shock was selected as a CS to discourage behavioral freezing and to provide an above-zero level of movement activity for evaluating the presence of conditioning. Three CS intensities and two US intensities were combined in a factorial design creating six separate experimental groups which received 50 paired trace conditioning trials at a CS-US interval of $8.1 \mathrm{sec}$. Three control groups were given 50 unpaired presentations of one of the CSs and the highest intensity US. All of the experimental groups showed the development of a bradycardia CR in the absence of a conditioned change in movement. During conditioning, HR and movement to CS onset varied together in both the experimental and control groups. Other examples of a close correspondence between HR and movement included some, but not all, of the preconditioning CS-alone responses, the URs, and shifts in baselines. The results suggested that although some nonassociatively based HR changes may have been tied to movement, the conditioned bradycardias in the experimental groups appeared to be relatively independent of movement.
\end{abstract}

Studies showing that changes in heart rate (HR) may covary directly with increases or decreases in skeletalmotor activity (Obrist et al., 1974) have led to the proposal (Obrist, 1981) that HR responses in a variety of behavioral situations may be tightly tied to movement through well-known (Rushmer, 1976) cardiacsomatomotor pathways within the central nervous system. Many of the studies showing a close correspondence between HR and movement have involved instrumental paradigms in which HR was measured while the subject received contingent reinforcement for changes in overt responding. The complexity of these paradigms and the absence of control procedures to evaluate the possible influences of nonassociative factors, such as sensitization and pseudoconditioning, have made it difficult to determine whether the HR changes accompanying movement were learned or nonspecific in nature.

That the relationship between learned HR responses and gross body movement may not be a simple one is suggested by the outcomes of aversive classical conditioning studies. A number of these studies have shown that although HR CRs (tachycardias as well as bradycardias) may at times covary with and be augmented in the presence of directionally similar changes in overt movement, the HR CRs are also seen in the absence of any movement (Bruner, 1969; Cohen, 1969; Fitzgerald, 1966; Martin \& Fitzgerald, 1980; Obrist et al., 1974; Smith \& Stebbins, 1965; Teyler, 1971). The latter outcomes sug-

This research was supported in part by a grant from the American Heart Association, Oregon Affiliate, and Grant HLO7332 from the National Heart, Lung, and Blood Institute. Requests for reprints should be sent to Robert Fitzgerald, Department of Medical Psychology L470, the Oregon Health Sciences University, 3181 SW Sam Jackson Park Road, Portland, OR 97201. gest that HR CRs and movement may not necessarily be tied together.

In addition to the lack of complete correspondence between HR CRs and movement, it should also be noted that few attempts have been made to determine whether the movement occurring with a HR CR was also conditioned. It would seem reasonable to assume that if conditioned modifications of HR are in fact linked to movement through common neural pathways, then the movement in question should become conditioned in a manner closely paralleling the conditioning of the HR CR. In most HR conditioning studies in which movement has also been measured, necessary control comparisons to establish that conditioning occurred have been made only for HR.

In a previous HR conditioning study (Martin \& Fitzgerald, 1980), tests for skeletal-motor learning in restrained rats provided evidence of conditioning of backmuscle EMG activity but not of gross body movement. The EMG CR, an increase in activity to the onset of an auditory CS, was associated with a bradycardia CR, suggesting that a state of immobility or behavioral freezing (Cohen \& Obrist, 1975) may have been learned. However, the relationship between the two CRs was less than perfect, since the EMG CR was restricted to the onset of the CS whereas the bradycardia CR was seen throughout the CS. Also, the EMG CR accompanied the HR CR in only one of several similarly treated groups, suggesting that the EMG conditioning effect was relatively weak. Nevertheless, even though weak, any tendency to freeze when the CS is presented could prevent potential gross-body-movement CRs from being seen.

An attempt was made in the present study to prevent freezing to CS onset by using a brief pulse of electric 
shock as the CS. The shock was sufficiently intense to elicit a burst of movement and a change in HR prior to the start of conditioning. It was reasoned that such a CS, in contrast to a more neutral auditory CS, would compete with freezing at the start of the CS-US interval, and at the same time serve as a signal for the forthcoming, more intense, shock US. Modification of the original reflex-movement response to the CS could then be used as an index of motor conditioning. Three different CSintensity shocks were examined in combination with two US-intensity shocks in a between-groups factorial design to determine if the possibility of parallel motor and HR conditioning varied with the magnitudes of the original reactions to the CS and US.

\section{METHOD}

\section{Subjects}

The subjects were 108 naive female 90 - to 120 -day-old LongEvans rats purchased from the Animal Care Department at the Oregon Health Sciences University. The subjects were maintained on a continuous light cycle to minimize hormonal changes associated with estrus. Food and water were available ad lib.

\begin{abstract}
Apparatus
To prevent extraneous sounds from reaching the rats, conditioning was carried out in an Industrial Acoustics Corporation environmental room into which continuous white noise $(75 \mathrm{~dB}$, re: .002 dynes $/ \mathrm{cm}^{2}$ ) was presented through a speaker. The rats were restrained in an inverted U-shaped Plexiglas cylinder manufactured by Narco Bio-Systems, Inc. Adjustable guillotine inserts at the front and back of the holder were positioned to hold the rats securely in place. The restrainer rested on a square Plexiglas plate which was supported at each corner by a stiff compression spring. Attached to the bottom surface of the place was an Astatic ceramic phonocartridge with a $6.5-\mathrm{cm}$-long metal rod mounted in place of the stylus assembly. Voltages generated by movement of the rod within the magnetic field of the cartridge windings were amplified by a Grass Model 5P1 dc amplifier and integrated into discrete pulses. The sensitivity of the system was set at a level that recorded all visible movements but not normal respiration. The ECG was recorded on a Grass Model 5 polygraph from two 20-ga hypodermic needles inserted under the skin on either side of the rat's chest. A lever-type microswitch was mounted above the polygraph pen assembly so that the switch was activated by the $R$ wave of each QRS complex. Heartbeats and movement pulses were accumulated in preselected time intervals on each trial and the totals encoded on a paper-tape punch.

There were three intensities of CS shock and two intensities of US shock generated by a Massey Dickinson constant-energy shocker. The CSs measured 60,120 , or $140 \mathrm{~V}$ dc and the USs 250 or $400 \mathrm{~V} \mathrm{dc}$. Each shock was a single $.5-\mathrm{msec}$ dc pulse delivered through two $1-\mathrm{cm}$-diam stainless steel electrodes placed $2 \mathrm{~cm}$ apart at the base of the rat's tail. The lowest CS shock was sufficiently intense to elicit tail flexion and slight vocalization. The timing of the CS and US shocks was controlled by transistorized logic modules.
\end{abstract}

\section{Procedure}

There were six experimental groups $(n=12$ each) randomly assigned to the cells of a $3 \times 2$ factorial design with one of the dimensions being the intensity of the CS and the other the intensity of the US. Three control groups $(n=12$ each) received unpaired presentations using one of the CSs and the highest intensity US. All groups were given a $30-\mathrm{min}$ adaptation period in the restrainer followed by six preconditioning trials with the CS alone. The experimental groups then received 50 trace conditioning trials with the single CS pulse of shock being paired with the single US pulse of shock at a CS-US interval of $8.1 \mathrm{sec}$. The control groups were given 50 unpaired presentations of the CS and US, with the CS being delivered 60,90 , or $120 \mathrm{sec}$ (mean $=90 \mathrm{sec}$ ) after the US. The mean intertrial interval during the preconditioning phase varied randomly among $180-, 210-$, or 240 -sec intervals (mean $=210 \mathrm{sec}$ ).

Heartbeats were recorded on each trial during an 8-sec baseline period located immediately prior to the delivery of the CS and during seven successive 2 -sec periods following the occurrence of the CS. Periods 1 through 4 were located during the 8.1-sec CS-US interval. Periods 5 through 7 occurred after the US. Heartbeats were not recorded during or for $100 \mathrm{msec}$ after the CS or US because of shock artifact. Difference scores were computed by converting the heartbeat totals to beats per minute (bpm) and then subtracting the baseline value from each of the 2 -sec values. Movement was recorded during the same intervals as $\mathrm{HR}$ except that recording continued throughout and immediately after the CS and US. Also, because almost all of the movement occurred during and shortly after the CS and US, Periods 3 and 4 during the CS-US interval were combined into a single period, as were the three post-US periods. Movement totals were converted to pulses/minute and then difference scores were computed just as for HR.

\section{RESULTS}

The HR and movement responses to the three CS intensities on the first CS-alone trial by itself and then averaged over successive two-trial blocks are shown in the left and right sides of Figure 1, respectively. Three groups were formed after an analysis of variance (ANOVA) showed that the US intensity and experimental/control factors (both dummy variables at this point) were not significant. The left of Figure 1 shows that on the first CSalone trial all three groups displayed mainly bradycardia. On the remaining trials, the HR responses of each group shifted to tachycardia, with response magnitude on the final trial block being directly related to CS intensity. The right of Figure 1 reveals that all groups showed a sharp increase in movement to CS onset and that, except for the low-intensity group on Trial 1 , movement then rapidly returned toward baseline. In keeping with what was seen for HR, movement to the CS generally increased over trials and was directly related to CS intensity. Separate three-way ANOVAs (CS intensity $\times$ trials $\times$ measurement intervals) provided significant CS intensity effects for $\operatorname{HR}[F(2,105)=3.50, \mathrm{p}<.05]$ and for movement $[\mathrm{F}(2,105)=4.80, \mathrm{p}<.01]$. There were also significant CS intensity $\times$ trial blocks $\times$ measurement intervals interactions for both responses $[\mathrm{HR}, \mathrm{F}(12,630)=3.57$, $\mathrm{p}<.01$; movement, $\mathrm{F}(8,420)=5.32, \mathrm{p}<.01]$, reflecting in each case the divergence of the groups over trials during the first measurement interval.

The HR and movement responses of the experimental and control groups to the CS during conditioning averaged across successive blocks of 10 trials are plotted in Figures 2 and 3, respectively. Three experimental groups were formed after it was determined by separate ANOVAs that US intensity did not significantly affect HR or movement responding to the CS. Inspection of Figure 2 reveals 


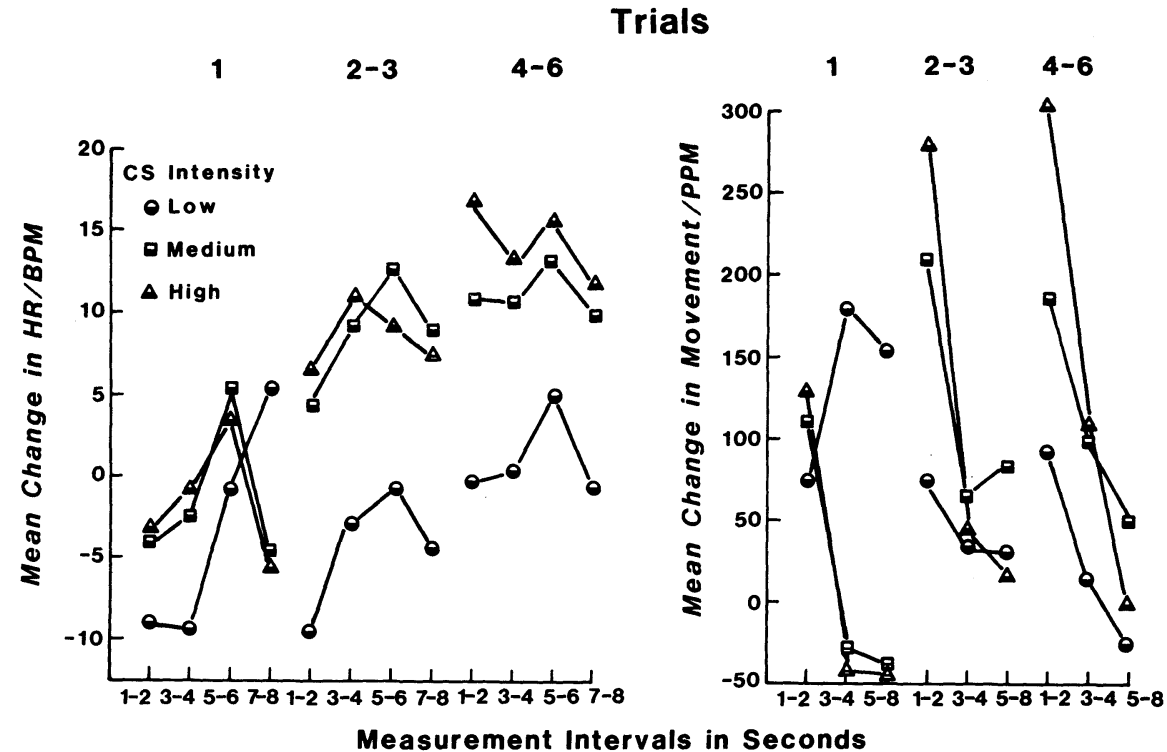

Figure 1. Original heart rate, in beats/minute (on the left), and movement, in pulses/minute (on the right), responses to the three CS intensities on the first preconditioning CS-alone trial and then on Trials 2-3, and Trials 4-6. For movement, the first measurement interval in each trial block was actually $2.1 \mathrm{sec}$ long.

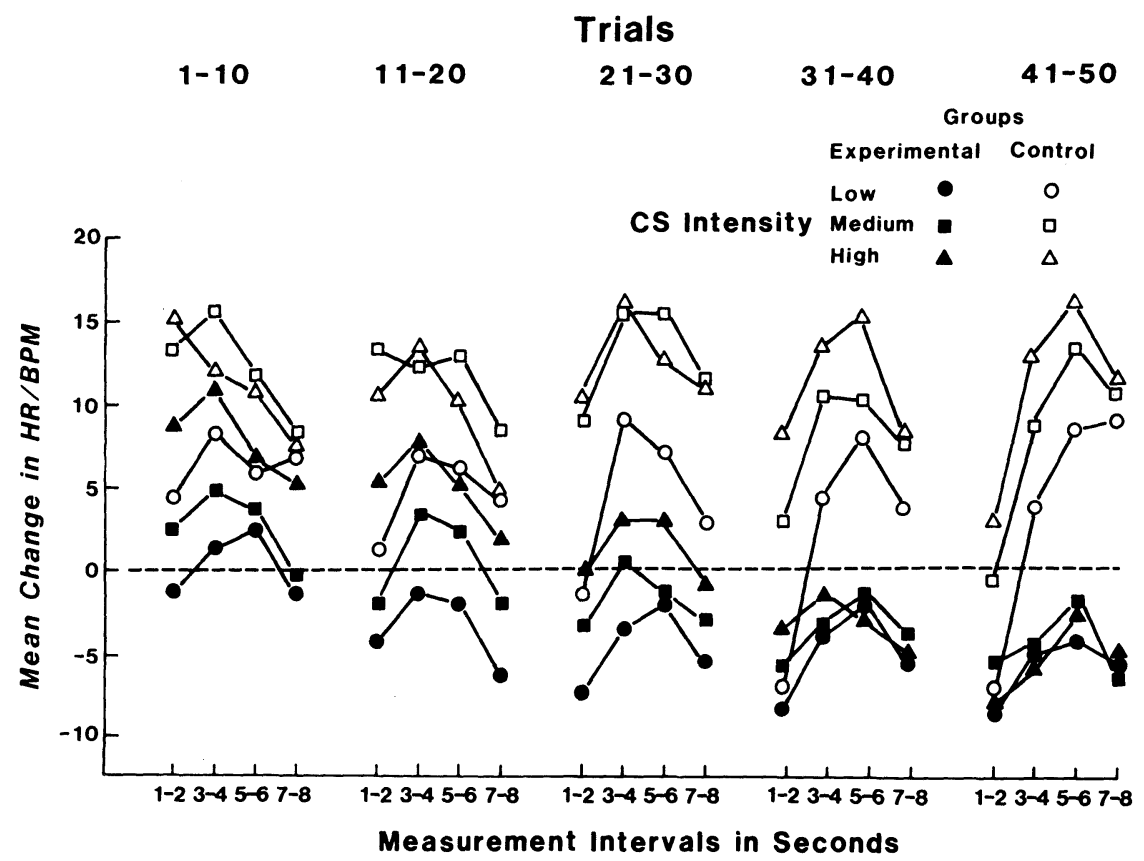

Figure 2. Heart rate responses of the experimental and control groups to the CS averaged over successive blocks of 10 conditioning trials.

that each of the experimental groups showed a gradual transition from tachycardia to bradycardia in all of the measurement intervals. The magnitudes of the bradycardia CRs were not affected by CS intensity. The HR responses of the control groups were mainly tachycardias, which generally varied in magnitude directly with CS intensity. However, all three control groups displayed a progres- sive loss of tachycardia in the first measurement interval, with the response in the low-intensity group actually becoming a bradycardia similar to that of the experimental groups. A $2 \times 3 \times 5 \times 4$ (experimental vs. control groups $\times$ CS intensity $\times$ trial blocks $\times$ measurement intervals) ANOVA gave a significant groups effect $[F(1,102)=$ $43.50, \mathrm{p}<.001]$ and a significant CS intensity effect 


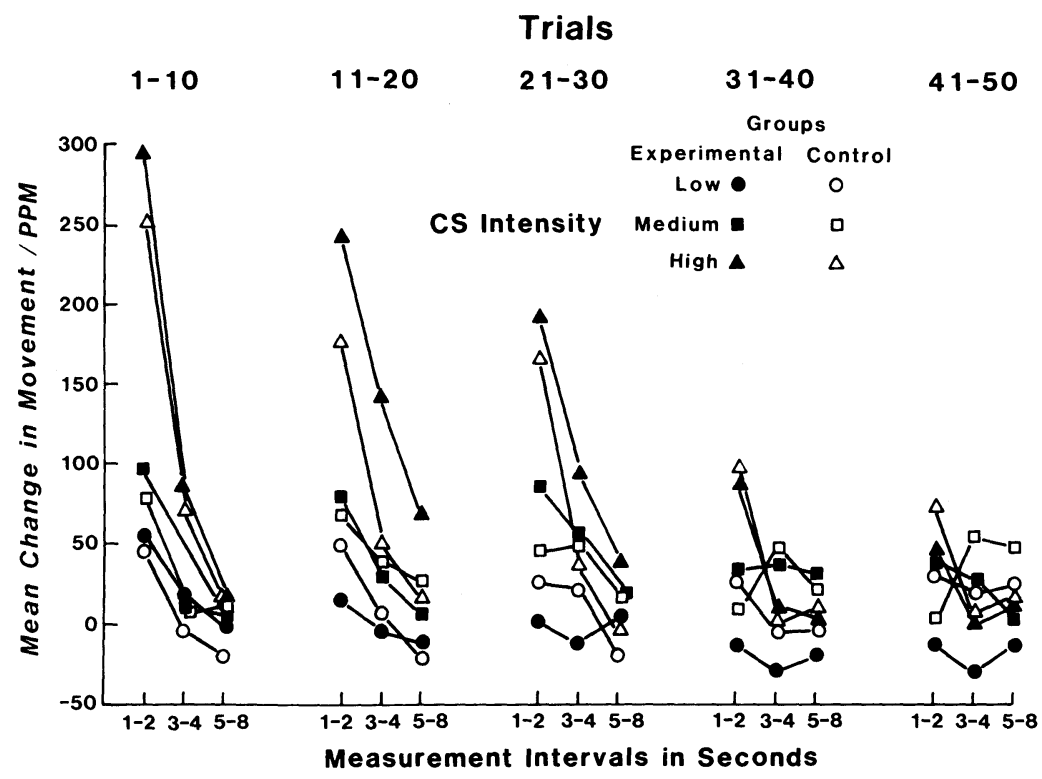

Figure 3. Movement responses of the experimental and control groups to the CS averaged over blocks of 10 conditioning trials. The first measurement interval in each trial block was actually 2.1 sec long.

$[F(2,102)=5.58, p<.01]$. Visually, the CS-intensity differences for the control groups appeared to be larger than those for the experimental groups, but the interaction reflecting this difference was not significant. Reliable conditioning of the bradycardia was indicated by a significant groups $\times$ trial blocks interaction $[\mathrm{F}(4,408)=$ $3.06, \mathrm{p}<.01$ ] and by a significant groups $\times$ trial blocks $\times$ measurement intervals interaction $[F(12,1224)=4.41$, $\mathrm{p}<.01]$.

Figure 3 indicates that the experimental and control groups showed similar movement responses to the CS throughout conditioning. At the start of conditioning there was an immediate increase in movement to CS onset, with movement then rapidly falling toward baseline. For both groups, the magnitude of the movement reactions decreased over trials. Prior to the decrease, response magnitude varied, in general, with CS intensity. A $2 \times 3 \times 5 \times 3$ (experimental vs. control $\times$ CS intensity $\times$ trial blocks $\times$ measurement intervals) ANOVA provided a significant CS intensity effect $[\mathrm{F}(2,102)=8.87, \mathrm{p}<.01]$ and a significant CS intensity $\times$ trial blocks $\times$ measurement intervals interaction $[F(16,816)=2.75, p<.01]$, reflecting the fact that the initial CS-intensity differences in the

Table 1

Pre-CS Base-Level Heart Rate and Movement in Blocks of 10 Conditioning Trials for the Combined Experimental and Control Groups

\begin{tabular}{cccccc}
\hline & \multicolumn{5}{c}{ Trials During Conditioning } \\
\cline { 2 - 6 } & $1-10$ & $11-20$ & $21-30$ & $31-40$ & $41-50$ \\
\hline $\begin{array}{c}\text { Heart rate } \\
\text { (bpm) } \\
\begin{array}{c}\text { Movement } \\
\text { (pulses/minute) }\end{array}\end{array}$ & 121 & 109 & 95 & 63 & 68 \\
\hline
\end{tabular}

first measurement interval diminished over the course of conditioning. There were no significant experimental versus control group differences, indicating that reliable conditioning of movement did not occur.

Pre-CS baseline HR and movement of all groups combined averaged over 10-trial blocks are shown in Table 1 . The groups were combined after it was determined that there were no significant baseline differences among the groups. Baseline HR and movement both decreased significantly over trials $[\mathrm{HR}, \mathrm{F}(4,420)=2.87, \mathrm{p}<.05$; movement, $\mathrm{F}(4,420)=4.01, \mathrm{p}<.01]$.

\section{DISCUSSION}

In general agreement with the results of other classical conditioning studies (e.g., Cohen, 1969; Fitzgerald, 1966; Martin \& Fitzgerald, 1980; Obrist et al., 1974), the present experiment showed that there was considerable independence between the HR CRs that developed to the shock CS and overt movement. All of the experimental groups, regardless of the intensity of the CS shocks, showed the development of a bradycardia CR in the absence of a conditioned change in overt movement. Thus, the pathways mediating the HR CRs were associatively based, in the sense that they were activated by CS/US pairings, whereas those responsible for movement were nonassociative in nature and not influenced by pairings. Although all three CS-shock levels were sufficiently intense to trigger major movement reactions accompanied by tachycardia, their use did not appear to facilitate the conditioning of movement or to prevent the conditioning of bradycardia. Finding that the pathways mediating the $\mathrm{HR}$ and movement changes during the CS-US interval were not the same is contrary to the view (Obrist, 1981) 
that HR CRs may necessarily be centrally linked to movement.

On the other hand, the failure to find that movement became conditioned does not mean that the HR CRs were completely independent of movement activity. Thus, all of the experimental groups showed a decrease in the amount of movement to CS onset over conditioning trials which paralleled, to some extent, the appearance of the HR CRs. This raises the possibility that short-latency components of the HR CRs may have been linked to the decreases in movement. The fact that the control groups also showed a tendency toward bradycardia (especially the low CS-intensity-control group) along with a loss of movement to CS onset suggests that the CS-onset reactions of all groups may have been controlled by nonassociative processes. Conceivably, major portions of the movement and HR changes elicited by the onset of the shock CSs were tied together as components of an unlearned defense or startle response (Cohen \& Obrist, 1975) which gradually habituated with exposure to the CS.

In an earlier study (Martin \& Fitzgerald, 1980), there was some evidence suggesting that bradycardia CRs in restrained rats might be linked to a skeletal-motor freezing reaction that becomes conditioned to the onset of the CS. Although the present findings do not rule out the possibility that such a linkage may exist under certain conditions, the linkage does not appear to be necessary, since bradycardia CRs occurred here to a shock CS that should have prevented freezing to CS onset.

It might be argued that the simultaneous occurrence of bradycardia and diminished movement shown by the experimental groups during the latter half of the CS-US interval provides evidence of cardiac-somatomotor coupling. However, this possibility is weakened by the fact that a highly similar low level of movement during that same period of time was associated with tachycardia in the control groups. If it is true that the CS-elicited movement responses of the experimental and control groups were controlled by the same nonassociative processes, and the absence of movement conditioning suggests that they were, then it becomes difficult to understand how those processes could be tied to bradycardia in the experimental groups as well as to tachycardia in the control groups. It seems more parsimonious to assume that the HR and movement changes in question were not tightly linked together.

There were several occasions in the current study when unlearned HR and movement seemed to be tightly coupled to each other. For example, the tachycardia and movement responses elicited on the CS-alone trials were, in general, both positively related to CS intensity and both responses increased in magnitude over the CS-alone trials. However, on the first CS-alone trial, HR and movement did not covary, since bradycardia was associated with a sizable increase in movement in all groups. During conditioning, the control groups tended to display tachycardia and movement increases, both of which varied directly with the intensity of the CS. Also, baseline HR and move- ment decreased together during conditioning. In general, these findings, as well as the CS-onset outcomes mentioned earlier, agree with other results (Obrist et al., 1974) showing that some kinds of HR and movement changes can covary in ways that suggest they share common pathways.

The successful development of a HR CR using shock as both the CS and US deserves some comment. The difference in the direction of the terminal CS-alone response (tachycardia) and the HR CR (bradycardia) suggests that the HR CR was not a modified version of the CS-alone response (Dykman, 1967; Fitzgerald \& Teyler, 1970). On the other hand, all of the groups showed a HR change on the first CS-alone trial that did, in fact, contain a short-latency decelerative component. Temporary, short-latency bradycardias have been observed previously in rats receiving electric shock for the first time (Fitzgerald, 1976), and these reactios have been found to be mediated mainly by increased vagal outflow (Fitzgerald, 1976). Thus, it is possible that the HR CR in the current study may have had vagal components in common with the original response to the CS.

Since the CS and US shocks were presented through the same electrodes to the same location on the rat's tail, the two stimuli could presumably be distinguished only on the basis of their intensity and/or their temporal order (i.e., CS first, US second). For a CR to develop under these conditions it could be assumed that an intensity discrimination was formed between the CS and US such that the weaker CS became a signal for the forthcoming stronger US. Alternatively, it might be that the CS and US intensity difference was not crucial and that the CS became a signal for the US simply because it was the first event in a closely spaced two-event sequence. The latter possibility would be consistent with a report (Prokasy, Williams, \& Clark, 1975) of GSR conditioning in humans using the same intensity auditory stimulus as the CS and US. In the present case, however, a single pulse of the same intensity shock to the rat's tail was employed as both the CS and US in an unpublished follow-up study, and no HR CR appeared. This suggests that an intensity discrimination between the CS and US shocks provided the basis for the bradycardia CRs that were seen in the current experiment.

\section{REFERENCES}

BRUNER, A. (1969). Reinforcement strength in classical conditioning of leg flexion, freezing, and heart rate in cats. Conditional Reflex, 4, 24-31.

CoHEN, D. H. (1969). Development of a vertebrate experimental model for cellular neurophysiological studies of learning. Conditional Reflex, 4, 61-80.

COHEN, D. H., \& OBRIST, P. A. (1975). Interactions between behavior and the cardiovascular system. Circulation Research, 37, 693-704.

Dykman, R. A. (1967). On the nature of classical conditioning. In C. C. Brown (Ed.), Methods of psychophysiology. Baltimore: Williams \& Wilkins.

FitzGerald, R. D. (1966). Some effects of partial reinforcement with shock on classically conditioned heart rate in dogs. American Journal of Psychology, 79, 242-249. 
FitzGerald, R. D. (1976). Involvement of vagal activity in the unconditioned heart-rate responses of restrained rats. Physiology \& Behavior, 17, 785-788.

Fitzgerald, R. D., \& TeYleR, T. J. (1970). Trace and delayed heartrate conditioning in rats as a function of US intensity. Journal of Comparative \& Physiological Psychology, 70, 242-253.

MARTIN, G. K., \& FitzGerald, R. D. (1980). Heart rate and somatomotor activity in rats during signalled escape and yoked classical conditioning. Physiology \& Behavior, 25, 519-526.

OBRIst, P. A. (1981). Cardiovascular psychophysiology: A perspective. New York: Plenum Press.

Obrist, P. A., Howard, J. L., Lawler, J. R., Galosy, R. A., Meyers, K. A., \& Gaebelein, C. J. (1974). The cardiac somatic interaction. In P. A. Obrist, A. H. Black, J. Brener, \& L. V. DiCara (Eds.), Cardiovascular psychophysiology: Current issues in response mechanisms, biofeedback and methodology (pp. 136-162). Chicago: Aldine.
Prokasy, W. F., Williams, W. C., \& Clark, C. G. (1975). Skin conductance response conditioning with $\mathrm{CS}$ intensities equal to and greater than UCS intensity. Memory \& Cognition, 3, 277-281.

Rushmer, R. F. (1976). Cardiovascular dynamics (4th ed). Philadelphia: Saunders.

Smith, O., \& Stebrins, W. C. (1965). Conditioned blood flow and heart rate in monkeys. Journal of Comparative \& Physiological Psychology, 59, 432-436.

TEYLER, T. J. (1971). Effects of restraint on heart rate conditioning in rats as a function of US location. Journal of Comparative and Physiological Pscyhology, 77, 31-37.

(Manuscript received May 18, 1985; revision accepted for publication September 10, 1985.)

\title{
Notice
}

\author{
Second National Traumatic Brain Injury Symposium \\ Baltimore, Maryland \\ April 17-18, 1986
}

The Second National Traumatic Brain Injury Symposium, “'The Road to Recovery," sponsored by the Speech-Communication Disorders Program, Maryland Institute for Emergency Medical Services Systems, University of Maryland Medical System, will be held April 17-18, 1986, in Baltimore, Maryland.

For further information, contact Roberta Schwartz, MIEMSS, 22 South Green Street, Baltimore, MD 21201. Phone: (301)528-6101. 